\title{
ПРИБЛИЖЕНИЯ ЛЮБОГО ПОРЯДКА АСИМПТОТИЧЕСКОГО РЕШЕНИЯ ТРЕХТЕМПОВОЙ ЛИНЕЙНО-КВАДРАТИЧНОЙ ЗАДАЧИ ОПТИМАЛЬНОГО УПРАВЛЕНИЯ МЕТОДОМ ПРЯМОЙ СХЕМЫ
}

\author{
М. А. Калашникова ${ }^{\star}$, Г. А. Курина ${ }^{* *}$ \\ ${ }^{*}$ Atos IT Solutions and Services \\ ${ }^{*}$ Воронежский государственный университет, \\ ${ }^{*}$ Воронежский экономико-правовой институт, \\ **Федеральный исследовательский центр "Информатика и управление» \\ Российской академии наук
}

Поступила в редакцию 29.09.2018 г.

\begin{abstract}
Аннотация. Построено приближение произвольного порядка асимптотического решения одного класса сингулярно возмущенных линейно-квадратичных задач оптимального управления. Переменные состояния содержат две группы быстрых переменных. В уравнениях для них перед производной стоят малый параметр и его квадрат. Используется метод прямой схемы, состоящий в подстановке постулируемого асимптотического разложения решения в условие задачи и построении серии более простых, чем исходная, задач для нахождения членов асимптотики. Искомое асимптотическое решение содержит пограничные функции четырех типов. Метод прямой схемы позволяет установить невозрастание значений минимизируемого функционала при использовании следующего приближения оптимального управления. Этот факт иллюстрируется в статье примером.

Ключевые слова: линейно-квадратичная задача управления, сингулярные возмущения, асимптотическое разложение, пограничные функции.

Annotation. The paper deals with constructing any order asymptotic solution of a class of singularly perturbed linear-quadratic optimal control problems. State variables contain two groups of fast variables. Equations for them have a small parameter or its square before the derivative. The direct scheme method has been used. This method consists of immediate substituting of a postulated asymptotic expansion of a solution into the problem condition and constructing a series of problems, which are simpler than the original one, for finding asymptotics terms. The sought for asymptotic solution contains boundary functions of four types. The direct scheme method allows us to establish the non-increasing of values of the minimized functional if we use a next asymptotic approximation to an optimal control. This fact is illustrated in the paper by an example.

Keywords: linear-quadratic control problem, singular perturbations, asymptotic expansion, boundary functions.
\end{abstract}

\section{ВВЕДЕНИЕ}

Линейно-квадратичные задачи являются как самостоятельным предметом изучения, так и хорошим инструментом при исследовании задач более сложной структуры. Задачам управления с быстрыми и медленными переменными посвящено большое число работ (см., например, обзоры [1], [2]). В основном изучаются системы с двухтемповыми пере-

(c) Калашникова М. А., Курина Г. А., 2018 менными. Однако на практике часто возникают системы с разнотемповыми быстрыми переменными. Краткий обзор публикаций, посвященных приложениям таких систем, приведен в [3]. При исследовании сингулярно возмущенных задач с разнотемповыми быстрыми переменными применяются как численные (см., например, [4]), так и асимптотические методы. Наиболее популярным среди асимптотических методов является метод пограничных функций [5]. Также применяется метод интегральных многообразий [6]. Ис- 


\section{М. А. Калашникова, Г. А. Курина}

пользованию асимптотических методов для изучения сингулярно возмущенных дискретных задач посвящен обзор [7]. Предельный переход решения задачи Коши для системы обыкновенных дифференциальных уравнений с малыми параметрами при производных при стремлении этих параметров к нулю к решению вырожденной задачи изучался в [8-10]. Асимптотический анализ сингулярно возмущенных задач управления с несколькими малыми параметрами обсуждался в статьях $[11,12]$, в которых отсутствуют ссылки на работы русскоязычных авторов в этом направлении.

При асимптотическом решении задач оптимального управления с параметрами возможны два подхода. Классический подход состоит в построении асимптотики задачи, получающейся из условий оптимальности управления. Другой способ - метод прямой схемы, в рамках которого в условие исходной задачи подставляется постулируемое асимптотическое разложение (в нашей статье это будет разложение погранслойного типа), а далее строится серия задач для нахождения членов асимптотики. Применение метода для асимптотического решения сингулярно возмущенных двухтемповых задач изложено в [13].

В настоящей статье при помощи метода прямой схемы будут найдены задачи меньшей размерности, чем исходная, решения которых являются членами асимптотического решения произвольного порядка линейно-квадратичной задачи оптимального управления с трехтемповыми переменными состояния. Асимптотика нулевого порядка для решения рассматриваемой задачи при помощи метода прямой схемы была построена ранее в [14].

Отметим, что к задачам с разнотемповыми быстрыми переменными путем замены переменных приводятся линейно-квадратичные задачи с дешевыми управлениями различных порядков малости (см., например, [15]). При этом в отличие от рассматриваемой в этой статье задачи получается сингулярно возмущенное уравнение состояния в критическом в смысле теории сингулярных возмущений случае.
Метод прямой схемы позволяет установить невозрастание значений минимизируемого функционала при использовании следующего приближения оптимального управления. Этот факт иллюстрируется в статье примером.

Далее $\varepsilon \geq 0$ - малый параметр, штрих означает транспонирование, $\langle$,$\rangle - скалярное$ произведение в соответствующих пространствах, $I_{k}$ - единичная матрица порядка $k$.

\section{1. ПОСТАНОВКА ЗАДАЧИ}

Рассмотрим следующую задачу

$$
\begin{gathered}
P_{\varepsilon}: J(u)= \\
=\frac{1}{2} \int_{0}^{T}(\langle w(t, \varepsilon), W(t, \varepsilon) w(t, \varepsilon)\rangle+ \\
+\langle u(t, \varepsilon), R(t, \varepsilon) u(t, \varepsilon)\rangle) d t \rightarrow \min , \\
\mathcal{E}(\varepsilon) \frac{d w(t, \varepsilon)}{d t}=A(t, \varepsilon) w(t, \varepsilon)+ \\
+B(t, \varepsilon) u(t, \varepsilon), \\
w(0, \varepsilon)=w^{0} .
\end{gathered}
$$

Здесь $t \in[0, T], \quad T>0$ - фиксировано, $w(t, \varepsilon)=\left(x(t, \varepsilon)^{\prime}, y(t, \varepsilon)^{\prime}, z(t, \varepsilon)^{\prime}\right)^{\prime}$, $w^{0}=\left(\left(x^{0}\right)^{\prime},\left(y^{0}\right)^{\prime},\left(z^{0}\right)^{\prime}\right)^{\prime}, \quad \quad x(t, \varepsilon) \in R^{n_{1}}$, $y(t, \varepsilon) \in R^{n_{2}}, \quad z(t, \varepsilon) \in R^{n_{3}}, \quad u(t, \varepsilon) \in R^{m}$, $\mathcal{E}(\varepsilon)=\operatorname{diag}\left(I_{n_{1}}, \varepsilon I_{n_{2}}, \varepsilon^{2} I_{n_{3}}\right)$, матрицы $W(t, \varepsilon)$, $R(t, \varepsilon), A(t, \varepsilon), B(t, \varepsilon)$ являются достаточно гладкими по своим аргументам, $W(t, \varepsilon)$, $R(t, \varepsilon)$ симметричны, $W(t, 0), R(t, 0)$ положительно определены при $t \in[0, T]$.

Запишем матрицу $A=A(t, \varepsilon)$ в блочной форме

$$
A=\left(\begin{array}{lll}
A_{11} & A_{12} & A_{13} \\
A_{12} & A_{22} & A_{23} \\
A_{13} & A_{23} & A_{33}
\end{array}\right) .
$$

Предположим, что выполнено условие устойчивости: действительные части собственных значений матриц $A_{33}$ и $\left(A_{22}-A_{23} A_{33}^{-1} A_{32}\right)$ отрицательны. 
Приближения любого порядка асимптотического решения...

\section{2. ДЕКОМПОЗИЦИЯ УСЛОВИЯ ЗАДАЧИ}

Будем искать решение исходной задачи $P_{\varepsilon}$ в виде разложения

$$
\begin{gathered}
v(t, \varepsilon)=\bar{v}(t, \varepsilon)+ \\
+\sum_{i=0}^{1}\left(\Pi_{i} v\left(\tau_{i}, \varepsilon\right)+Q_{i} v\left(\sigma_{i}, \varepsilon\right)\right),
\end{gathered}
$$

где $v(t, \varepsilon) \stackrel{i=0}{=}\left(w(t, \varepsilon)^{\prime}, u(t, \varepsilon)^{\prime}\right)^{\prime}$,

$\bar{v}(t, \varepsilon)=\sum_{j \geq 0} \varepsilon^{j} \bar{v}_{j}(t), \quad \Pi_{i} v\left(\tau_{i}, \varepsilon\right)=\sum_{j \geq 0} \varepsilon^{j} \Pi_{i j} v\left(\tau_{i}\right)$, $Q_{i} v\left(\sigma_{i}, \varepsilon\right)=\sum_{j \geq 0} \varepsilon^{j} Q_{i j} v\left(\sigma_{i}\right), \quad \tau_{i}=t / \varepsilon^{i+1}$, $\sigma_{i}=(t-T) / \varepsilon^{i+1}, \quad i=0,1, \bar{v}_{j}(t)$ - регулярные функции, $\Pi_{i j} v\left(\tau_{i}\right)$ - пограничные функции экспоненциального типа в окрестности $t=0$, $Q_{i j} v\left(\sigma_{i}\right)$ - пограничные функции экспоненциального типа в окрестности $t=T$.

Введем обозначения $E_{1}=\operatorname{diag}\left(I_{n_{1}}, 0,0\right)$, $E_{2}=\operatorname{diag}\left(0, I_{n_{2}}, 0\right), E_{3}=\operatorname{diag}\left(0,0, I_{n_{3}}\right)$,

$$
\begin{aligned}
F(v, t, \varepsilon)= & 1 / 2(\langle w(t, \varepsilon), W(t, \varepsilon) w(t, \varepsilon)\rangle+ \\
& +\langle u(t, \varepsilon), R(t, \varepsilon) u(t, \varepsilon)\rangle), \\
\Phi(v, t, \varepsilon)= & A(t, \varepsilon) w(t, \varepsilon)+ \\
& +B(t, \varepsilon) u(t, \varepsilon) .
\end{aligned}
$$

Разложение произвольной функции $\omega=\omega(\varepsilon)$ в ряд по целым неотрицательным степеням $\varepsilon$ будем представлять в виде $\omega(\varepsilon)=\sum_{j \geq 0} \varepsilon^{j} \omega_{j}=\{\omega\}_{n-1}+\varepsilon^{n}[\omega]_{n}+\alpha\left(\varepsilon^{n+1}\right)$, где $\{\omega\}_{n-1}=\sum_{j=0}^{n-1} \varepsilon^{j} \omega_{j},[\omega]_{n}=\omega_{n}$, а $\alpha\left(\varepsilon^{n+1}\right)$ - сумма членов разложения порядка $\varepsilon^{n+1}$ и выше.

Подставим разложение (4) в уравнение состояния (2). Приравнивая коэффициенты при одинаковых степенях $\varepsilon$, отдельно зависящие от $t, \tau_{i}, \sigma_{i}, i=0,1$, получаем уравнения

$$
\begin{gathered}
E_{1} \frac{d \bar{w}_{j}(t)}{d t}+E_{2} \frac{d \bar{w}_{j-1}(t)}{d t}+ \\
+E_{3} \frac{d \bar{w}_{j-2}(t)}{d t}=A_{0}(t) \bar{w}_{j}(t)+ \\
+B_{0}(t) \bar{u}_{j}(t)+\left[\widehat{\bar{\Phi}}_{(j-1)}(t, \varepsilon)\right]_{j}, \\
\left(E_{1}+E_{2}\right) \frac{d \Pi_{0 j} w\left(\tau_{0}\right)}{d \tau_{0}}+ \\
+E_{3} \frac{d \Pi_{0(j-1)} w\left(\tau_{0}\right)}{d \tau_{0}}=
\end{gathered}
$$

$$
\begin{gathered}
=E_{1}\left[\hat{\Pi}_{0(j-1)} \Phi\left(\tau_{0}, \varepsilon\right)\right]_{j-1}+ \\
+\left(E_{2}+E_{3}\right)\left(A_{0}(0) \Pi_{0 j} w\left(\tau_{0}\right)+\right. \\
\left.+B_{0}(0) \Pi_{0 j} u\left(\tau_{0}\right)+\left[\hat{\Pi}_{0(j-1)} \Phi\left(\tau_{0}, \varepsilon\right)\right]_{j}\right) \\
\left(E_{1}+E_{2}\right) \frac{d Q_{0 j} w\left(\sigma_{0}\right)}{d \sigma_{0}}+ \\
+E_{3} \frac{d Q_{0(j-1)} w\left(\sigma_{0}\right)}{d \sigma_{0}}= \\
=E_{1}\left[\hat{Q}_{0(j-1)} \Phi\left(\sigma_{0}, \varepsilon\right)\right]_{j-1}+ \\
+\left(E_{2}+E_{3}\right)\left(A_{0}(T) Q_{0 j} w\left(\sigma_{0}\right)+\right. \\
\left.+B_{0}(T) Q_{0 j} u\left(\sigma_{0}\right)+\left[\hat{Q}_{0(j-1)} \Phi\left(\sigma_{0}, \varepsilon\right)\right]_{j}\right), \\
\frac{d \Pi_{1 j} w\left(\tau_{1}\right)}{d \tau_{1}}=E_{1}\left[\hat{\Pi}_{1(j-2)} \Phi\left(\tau_{1}, \varepsilon\right)\right]_{j-2}+ \\
+E_{2}\left[\hat{\Pi}_{1(j-1)} \Phi\left(\tau_{1}, \varepsilon\right)\right]_{j-1}+ \\
+E_{3}\left(A_{0}(0) \Pi_{1 j} w\left(\tau_{1}\right)\right. \\
\left.+B_{0}(0) \Pi_{1 j} u\left(\tau_{1}\right)+\left[\hat{\Pi}_{1(j-1)} \Phi\left(\tau_{1}, \varepsilon\right)\right]_{j}\right), \\
\frac{d Q_{1 j} w\left(\sigma_{1}\right)}{d \sigma_{1}}=E_{1}\left[\hat{Q}_{1(j-2)} \Phi\left(\sigma_{1}, \varepsilon\right)\right]_{j-2}+ \\
+E_{2}\left[\hat{Q}_{1(j-1)} \Phi\left(\sigma_{1}, \varepsilon\right)\right]_{j-1}+ \\
+E_{3}\left(A_{0}(T) Q_{1 j} w\left(\sigma_{1}\right)\right. \\
\left.+B_{0}(T) Q_{1 j} u\left(\sigma_{1}\right)+\left[\hat{Q}_{1(j-1)} \Phi\left(\sigma_{1}, \varepsilon\right)\right]_{j}\right) .
\end{gathered}
$$

Здесь $\widehat{\bar{\Phi}}_{j}(t, \varepsilon), \hat{\Pi}_{i j} \Phi\left(\tau_{i}, \varepsilon\right), \hat{Q}_{i j} \Phi\left(\sigma_{i}, \varepsilon\right)$ являются значениями функции $\Phi$ при $v=\tilde{\bar{v}}(t, \varepsilon), \quad v=\tilde{\Pi}_{i j} v\left(\tau_{i}, \varepsilon\right) \quad$ и $\quad t=\varepsilon^{j+1} \tau_{i}$, $v=\tilde{Q}_{i j} v\left(\sigma_{i}, \varepsilon\right)$ и $t=T+\varepsilon^{j+1} \sigma_{i}, \quad i=0,1$, соответственно. Функции с отрицательными индексами считаются нулевыми.

В силу убывания пограничных функций на бесконечности из уравнений (6)-(9) при $j=0,(8),(9)$ при $j=1$, следуют равенства

$$
\begin{aligned}
& \Pi_{00} x\left(\tau_{0}\right)=0, \Pi_{10} x\left(\tau_{1}\right)=\Pi_{11} x\left(\tau_{1}\right)=0, \\
& Q_{00} x\left(\sigma_{0}\right)=0, Q_{10} x\left(\sigma_{1}\right)=Q_{11} x\left(\sigma_{1}\right)=0, \\
& \Pi_{10} y\left(\tau_{1}\right)=0, Q_{10} y\left(\sigma_{1}\right)=0 .
\end{aligned}
$$

Принимая во внимание последние соотношения, из (3) получаем начальные условия для членов разложения

$$
\begin{gathered}
\bar{x}_{0}(0)=x^{0}, \bar{x}_{1}(0)+\Pi_{01} x(0)=0, \\
\bar{x}_{j}(0)+\Pi_{0 j} x(0)+\Pi_{1 j} x(0)=0, j \geq 2, \\
\bar{y}_{0}(0)+\Pi_{00} y(0)=y^{0},
\end{gathered}
$$




$$
\begin{gathered}
\bar{y}_{j}(0)+\Pi_{0 j} y(0)+\Pi_{1 j} y(0)=0, j \geq 1, \\
\bar{z}_{j}(0)+\Pi_{0 j} z(0)+\Pi_{1 j} z(0)=\left\{\begin{array}{c}
z^{0}, j=0, \\
0, j \geq 1 .
\end{array}\right.
\end{gathered}
$$

Подставляя разложение (4) в (1), представим подынтегральное выражение в виде суммы слагаемых по степеням малого параметра, отдельно зависящих от $t, \tau_{i}, \sigma_{i}, i=0,1$, причем в интегральных выражениях от $\tau_{i}, \sigma_{i}$, $i=0,1$, перейдем к интегрированию по бесконечным промежуткам $[0,+\infty)$ и $(-\infty, 0]$ соответственно. В результате критерий качества можно записать в виде

$$
J(u)=\sum_{j \geq 0} \varepsilon^{j} J_{j} .
$$

В [15] методом прямой схемы получено приближение нулевого порядка рассматриваемой задачи (1)-(3). Далее будут приведены задачи оптимального управления для нахождения членов асимптотики произвольного порядка.

\section{3. ПРИБЛИЖЕНИЯ ВЫСШИХ ПОРЯДКОВ}

Введем обозначения

$$
\begin{gathered}
\quad \varsigma(w, \psi, t, \varepsilon)=W(t, \varepsilon) w(t, \varepsilon)- \\
-A(t, \varepsilon)^{\prime} \psi(t, \varepsilon), \varrho(w, \psi, t, \varepsilon)= \\
=R(t, \varepsilon) w(t, \varepsilon)-B(t, \varepsilon)^{\prime} \psi(t, \varepsilon) .
\end{gathered}
$$

Задача $\bar{P}_{j}$ для определения регулярных функций $\bar{v}_{j}(t), t \in[0, T], j=\overline{0, n-1}$, заключается в минимизации функционала

$$
\begin{gathered}
\bar{J}_{j}\left(\bar{u}_{j}\right)= \\
=\left\langle\bar{w}_{j}(T), E_{1}\left(Q_{0(j-1)} \psi(0)+Q_{1(j-2)} \psi(0)\right)\right\rangle+ \\
+\int_{0}^{T}\left(\left\langle\bar{w}_{j}(t), 1 / 2 W_{0}(t) \bar{w}_{j}(t)+\left[\hat{\bar{\zeta}}_{j-1}(t, \varepsilon)\right]_{j}-\right.\right. \\
\left.-E_{2} d \bar{\psi}_{j-1}(t) / d t-E_{3} d \bar{\psi}_{j-2}(t) / d t\right\rangle+ \\
\left.+\left\langle\bar{u}_{j}(t), 1 / 2 R_{0}(t) \bar{u}_{j}(t)+\left[\hat{\bar{\varrho}}_{j-1}(t, \varepsilon)\right]_{j}\right\rangle\right) d t
\end{gathered}
$$

на траекториях системы (5) при условии (10). Символами $\hat{\bar{\zeta}}_{j-1}(t, \varepsilon), \hat{\bar{\varrho}}_{j-1}(t, \varepsilon)$ обозначены значения функций $\varsigma, \varrho$ при $v=\hat{\bar{v}}_{j}(t, \varepsilon)$, $\psi=\widehat{\bar{\psi}}_{j}(t, \varepsilon), \bar{\psi}_{j}(t)$ - сопряженные переменные в задачах оптимального управления $\bar{P}_{j}$, $j=\overline{0, n-1}$.
Задача $\Pi_{0 j} P$ для нахождения пограничных функций $\Pi_{0 j} v\left(\tau_{0}\right), \tau_{0} \in[0,+\infty), j=0, n-1$, заключается в минимизации функционала

$$
\begin{gathered}
\Pi_{0 j} J\left(\Pi_{0 j} u\right)= \\
=\int_{0}^{+\infty}\left(\left\langle\Pi_{0 j} w\left(\tau_{0}\right), 1 / 2 W_{0}(0) \Pi_{0 j} w\left(\tau_{0}\right)+\right.\right. \\
+\left[\hat{\Pi}_{0(j-1)} \varsigma\left(\tau_{0}, \varepsilon\right)\right]_{j}- \\
\left.-E_{3} d \Pi_{0(j-1)} \psi\left(\tau_{0}\right) / d \tau_{0}\right\rangle+ \\
+\left\langle\Pi_{0 j} u\left(\tau_{0}\right), 1 / 2 R_{0}(0) \Pi_{0 j} u\left(\tau_{0}\right)+\right. \\
\left.\left.+\left[\hat{\Pi}_{0(j-1)} \varrho\left(\tau_{0}, \varepsilon\right)\right]_{j}\right\rangle\right) d \tau_{0}
\end{gathered}
$$

на траекториях системы (6) при условиях $E_{1} \Pi_{0 j} w(+\infty)=0$ и (11), где символами $\hat{\Pi}_{0(j-1)} \varsigma\left(\tau_{0}, \varepsilon\right), \hat{\Pi}_{0(j-1)} \varrho\left(\tau_{0}, \varepsilon\right)$ обозначены значения функций $\varsigma$, $\varrho$ при $v=\tilde{\Pi}_{0(j-1)} v\left(\tau_{0}, \varepsilon\right)$, $\psi=\left(\varepsilon E_{1}+E_{2}+E_{3}\right) \tilde{\Pi}_{0(j-1)} \psi\left(\tau_{0}, \varepsilon\right), \quad t=\varepsilon \tau_{0}$, $\Pi_{0 j} \psi\left(\tau_{0}\right)$ - сопряженные переменные в задачах оптимального управления $\Pi_{0 j} P, j=\overline{0, n-1}$.

Задача $Q_{0 j} P$ для нахождения пограничных функций $Q_{0 j} v\left(\sigma_{0}\right), \quad \sigma_{0} \in(-\infty, 0], \quad j=\overline{0, n-1}$, состоит в минимизации функционала

$$
\begin{gathered}
Q_{0 j} J\left(Q_{0 j} u\right)= \\
=\left\langle Q_{0 j} w(0), E_{2}\left(\bar{\psi}_{j}(T)+Q_{1(j-1)} \psi(0)\right)\right\rangle+ \\
+\int_{-\infty}^{0}\left(\left\langleQ_{0 j} w\left(\sigma_{0}\right), 1 / 2 W_{0}(T) Q_{0 j} w\left(\sigma_{0}\right)+\right.\right. \\
+\left[\hat{Q}_{0(j-1)} \varsigma\left(\sigma_{0}, \varepsilon\right)\right]_{j}- \\
\left.-E_{3} d Q_{0(j-1)} \psi\left(\sigma_{0}\right) / d \sigma_{0}\right\rangle+ \\
+\left\langle Q_{0 j} u\left(\sigma_{0}\right), 1 / 2 R_{0}(T) Q_{0 j} u\left(\sigma_{0}\right)+\right. \\
\left.\left.+\left[\hat{Q}_{0(j-1)} \varrho\left(\sigma_{0}, \varepsilon\right)\right]_{j}\right\rangle\right) d \sigma_{0}
\end{gathered}
$$

на траекториях системы (7) при условии $\left(E_{1}+E_{2}\right) Q_{0 j} w(-\infty)=0$, где символами $\hat{Q}_{0(j-1)} \varsigma\left(\sigma_{0}, \varepsilon\right), \hat{Q}_{0(j-1)} \varrho\left(\sigma_{0}, \varepsilon\right)$ обозначены значения функций $\varsigma, \varrho$ при $v=\tilde{Q}_{0(j-1)} v\left(\sigma_{0}, \varepsilon\right)$, $\psi=\left(\varepsilon E_{1}+E_{2}+E_{3}\right) \tilde{Q}_{0(j-1)} \psi\left(\sigma_{0}, \varepsilon\right), \quad t=T+\varepsilon \sigma_{0}$, $Q_{0 j} \psi\left(\sigma_{0}\right)$ - сопряженные переменные в линейно-квадратичных задачах оптимального управления $Q_{0 j} P, j=\overline{0, n-1}$.

Задача $\Pi_{1 j} P$ для нахождения пограничных функций $\Pi_{1 j} v\left(\tau_{1}\right), \tau_{1} \in[0,+\infty), j=0, n-1$, состоит в минимизации функционала 
Приближения любого порядка асимптотического решения...

$$
\begin{gathered}
\Pi_{1 j} J\left(\Pi_{1 j} u\right)= \\
=\int_{0}^{+\infty}\left(\left\langle\Pi_{1 j} w\left(\tau_{1}\right), 1 / 2 W_{0}(0) \Pi_{1 j} w\left(\tau_{1}\right)+\right.\right. \\
\left.+\left[\hat{\Pi}_{1(j-1)} \varsigma\left(\tau_{1}, \varepsilon\right)\right]_{j}\right\rangle+ \\
+\left\langle\Pi_{1 j} u\left(\tau_{1}\right), 1 / 2 R_{0}(0) \Pi_{1 j} u\left(\tau_{1}\right)+\right. \\
\left.\left.+\left[\hat{\Pi}_{1(j-1)} \varrho\left(\tau_{1}, \varepsilon\right)\right]_{j}\right\rangle\right) d \tau_{1}
\end{gathered}
$$

на траекториях системы (8) при условиях $\left(E_{1}+E_{2}\right) \Pi_{1 j} w(+\infty)=0$ и (12), где символами $\hat{\Pi}_{1(j-1)} \varsigma\left(\tau_{1}, \varepsilon\right), \hat{\Pi}_{1(j-1)} \varrho\left(\tau_{1}, \varepsilon\right)$ обозначены соответственно значения функции $\zeta, \varrho$ при $v=\tilde{\Pi}_{1(j-1)} v\left(\tau_{1}, \varepsilon\right)$,

$\psi=\left(\varepsilon^{2} E_{1}+\varepsilon E_{2}+E_{3}\right) \tilde{\Pi}_{1(j-1)} \psi\left(\tau_{1}, \varepsilon\right), \quad t=\varepsilon^{2} \tau_{1}$, $\Pi_{1 j} \psi\left(\tau_{1}\right)$ - сопряженные переменные в задачах $\Pi_{1 j} P, j=\overline{0, n-1}$.

Задача $Q_{1 j} P$ для нахождения пограничных функций $Q_{1 j} v\left(\sigma_{1}\right), \sigma_{1} \in(-\infty, 0], j=\overline{0, n-1}$, состоит в минимизации функционала

$$
\begin{gathered}
Q_{1 j} J\left(Q_{1 j} u\right)= \\
=\left\langle Q_{1 j} w(0), E_{3}\left(\bar{\psi}_{j}(T)+Q_{1 j} \psi(0)\right)\right\rangle+ \\
+\int_{-\infty}^{0}\left(\left\langleQ_{1 j} w\left(\sigma_{1}\right), 1 / 2 W_{0}(T) Q_{1 j} w\left(\sigma_{1}\right)+\right.\right. \\
\left.+\left[\hat{Q}_{1(j-1)} \varsigma\left(\sigma_{1}, \varepsilon\right)\right]_{j}\right\rangle+ \\
+\left\langle Q_{1 j} u\left(\sigma_{1}\right), 1 / 2 R_{0}(T) Q_{1 j} u\left(\sigma_{1}\right)+\right. \\
\left.\left.+\left[\hat{Q}_{1(j-1)} \varrho\left(\sigma_{1}, \varepsilon\right)\right]_{j}\right\rangle\right) d \sigma_{1}
\end{gathered}
$$

на траекториях системы (9) при условии $Q_{1 j} w(-\infty)=0$, где символами $\hat{Q}_{1(j-1)} \varsigma\left(\sigma_{1}, \varepsilon\right), \hat{Q}_{1(j-1)} \varrho\left(\sigma_{1}, \varepsilon\right)$ обозначены значения функции $\varsigma, \varrho$ при $v=\tilde{Q}_{1(j-1)} v\left(\sigma_{1}, \varepsilon\right)$, $\psi=\left(\varepsilon^{2} E_{1}+\varepsilon E_{2}+E_{3}\right) \tilde{Q}_{1(j-1)} \psi\left(\sigma_{1}, \varepsilon\right)$,

$t=T+\varepsilon^{2} \sigma_{1}, Q_{1 j} \psi\left(\sigma_{1}\right)$ - сопряженные переменные в задачах $Q_{1 j} P, j=\overline{0, n-1}$.

Замечание. Из уравнений (6)-(9) следует, что $\quad E_{1} \Pi_{i n} w\left(\tau_{i}\right), \quad E_{1} Q_{\text {in }} w\left(\sigma_{i}\right), \quad i=0,1$, $E_{1} \Pi_{1(n+1)} w\left(\tau_{1}\right), \quad E_{1} Q_{1(n+1)} w\left(\sigma_{1}\right), \quad E_{2} \Pi_{1 n} w\left(\tau_{1}\right)$, $E_{2} Q_{1 n} w\left(\sigma_{1}\right)$ являются известными функциями после решения задач $\Pi_{i j} P, Q_{i j} P, i=0,1$, $j=\overline{0, n-1}$.

Задача $P_{\varepsilon}$ однозначно разрешима при $\varepsilon>0$. Оптимальное управление $u(t, \varepsilon)$ удовлетворяет условию

$$
u(t, \varepsilon)=R(t, \varepsilon)^{-1} B(t, \varepsilon)^{\prime} \xi(t, \varepsilon),
$$

где сопряженная переменная $\xi(t, \varepsilon)=\left(\zeta(t, \varepsilon)^{\prime}, \eta(t, \varepsilon)^{\prime}, \theta(t, \varepsilon)^{\prime}\right)^{\prime}$ - решение задачи

$$
\begin{gathered}
\mathcal{E}(\varepsilon) \frac{d \xi(t, \varepsilon)}{d t}=W(t, \varepsilon) w(t, \varepsilon)- \\
-A(t, \varepsilon)^{\prime} \xi(t, \varepsilon), \\
\xi(T, \varepsilon)=0 .
\end{gathered}
$$

Представим $\xi(t, \varepsilon)$ в виде асимптотической суммы

$$
\begin{gathered}
\xi(t, \varepsilon)=\bar{\xi}(t, \varepsilon)+ \\
+\sum_{i=0}^{1}\left(\Pi_{i} \xi\left(\tau_{i}, \varepsilon\right)+Q_{i} \xi\left(\sigma_{i}, \varepsilon\right)\right),
\end{gathered}
$$

каждое слагаемое которой раскладывается по степеням малого параметра с пограничными функциями экспоненциального типа аналогично (4).

Дополнительно введем функции $f(w, \xi, t, \varepsilon)=W(t, \varepsilon) w(t, \varepsilon)-A(t, \varepsilon)^{\prime} \xi(t, \varepsilon)$, $g(u, \xi, t, \varepsilon)=B(t, \varepsilon)^{\prime} \xi(t, \varepsilon)-R(t, \varepsilon) u(t, \varepsilon)$.

Обозначения $\hat{\bar{f}}_{j}(t, \varepsilon), \quad \hat{\Pi}_{i j} f\left(\tau_{i}, \varepsilon\right)$, $\hat{Q}_{i j} f\left(\sigma_{i}, \varepsilon\right), \hat{\bar{g}}_{j}(t, \varepsilon), \hat{\Pi}_{i j} g\left(\tau_{i}, \varepsilon\right), \hat{Q}_{i j} g\left(\sigma_{i}, \varepsilon\right)$ аналогичны соответствующим обозначениям для функций $\Phi, \varsigma, \varrho$.

Подставляя разложения (4), (17) в (14)(16), приравнивая коэффициенты при одинаковых степенях малого параметра, отдельно зависящие от $t, \tau_{i}, \sigma_{i}, i=0,1$, получаем соотношения

$$
\begin{gathered}
B_{0}(t)^{\prime} \bar{\xi}_{j}-R_{0}(t) \bar{u}_{j}+\left[\hat{\bar{g}}_{j-1}(t, \varepsilon)\right]_{j}=0 \\
E_{1} \frac{d \bar{\xi}_{j}}{d t}+E_{2} \frac{d \bar{\xi}_{j-1}}{d t}+E_{3} \frac{d \bar{\xi}_{j-2}}{d t}= \\
=W_{0}(t) \bar{w}_{j}-A_{0}(t)^{\prime} \bar{\xi}_{j}+\left[\widehat{\bar{f}}_{j-1}(t, \varepsilon)\right]_{j}, \\
B_{0}(0)^{\prime} \Pi_{i j} \xi-R_{0}(0) \Pi_{i j} u+ \\
+\left[\hat{\Pi}_{i(j-1)} g\left(\tau_{i}, \varepsilon\right)\right]_{j}=0 \\
E_{1} \frac{d \Pi_{i j}}{d \tau_{i}}+E_{2} \frac{d \Pi_{i(j-1)}}{d \tau_{i}}+E_{3} \frac{d \Pi_{i(j-2)}}{d \tau_{i}}= \\
=W_{0}(0) \Pi_{i(j-i-1)} w-A_{0}(0)^{\prime} \Pi_{i(j-i-1)} \xi+ \\
+\left[\hat{\Pi}_{i(j-i-2)} f\left(\tau_{i}, \varepsilon\right)\right]_{j-i-1},
\end{gathered}
$$


М. А. Калашникова, Г. А. Курина

$$
\begin{gathered}
B_{0}(T)^{\prime} Q_{i j} \xi-R_{0}(T) Q_{i j} u+ \\
+\left[\hat{Q}_{i(j-1)} g\left(\sigma_{i}, \varepsilon\right)\right]_{j}=0, \\
E_{1} \frac{d Q_{i j} \xi}{d \sigma_{i}}+E_{2} \frac{d Q_{i(j-1)} \xi}{d \sigma_{i}}+E_{3} \frac{d Q_{i(j-2)} \xi}{d \sigma_{i}}= \\
=W_{0}(T) Q_{i(j-i-1)} w-A_{0}(T)^{\prime} Q_{i(j-i-1)} \xi+ \\
+\left[\hat{Q}_{i(j-i-2)} f\left(\sigma_{i}, \varepsilon\right)\right]_{j-i-1}, \\
\bar{\xi}_{j}(T)+Q_{0 j} \xi(0)+Q_{1 j} \xi(0)=0 .
\end{gathered}
$$

Сформулируем теоремы о связи между условиями оптимальности управления для задач $\bar{P}_{j} \Pi_{i j} P, Q_{i j} P, i=0,1$, и соотношениями для членов асимптотики (4), (17).

Теорема 1. Краевые задачи, полученные из условий оптимальности управления для задач $\bar{P}_{j}, \Pi_{i j} P, Q_{i j} P, i=0,1$, совпадают coomветственно с задачами для

$$
\begin{gathered}
\left(\bar{v}_{j}(t), \bar{\xi}_{j}(t)\right), \\
\left(\Pi_{0 j} v\left(\tau_{0}\right), E_{1} \Pi_{0(j+1)} \xi\left(\tau_{0}\right)+\right. \\
\left.+\left(E_{2}+E_{3}\right) \Pi_{0 j} \xi\left(\tau_{0}\right)\right), \\
\left(Q_{0 j} v\left(\sigma_{0}\right), E_{1} Q_{0(j+1)} \xi\left(\sigma_{0}\right)+\right. \\
\left.+\left(E_{2}+E_{3}\right) Q_{0 j} \xi\left(\sigma_{0}\right)\right), \\
\left(\Pi_{1 j} v\left(\tau_{1}\right), E_{1} \Pi_{1(j+2)} \xi\left(\tau_{1}\right)+\right. \\
\left.+E_{2} \Pi_{1(j+1)} \xi\left(\tau_{1}\right)+E_{3} \Pi_{1 j} \xi\left(\tau_{1}\right)\right), \\
\left(Q_{1 j} v\left(\sigma_{1}\right), E_{1} Q_{1(j+2)} \xi\left(\sigma_{1}\right)+\right. \\
\left.+E_{2} Q_{1(j+1)} \xi\left(\sigma_{1}\right)+E_{3} Q_{1 j} \xi\left(\sigma_{1}\right)\right)
\end{gathered}
$$

из асимптотики (4), (17) решения задачи (2), (3), (14)-(16), вытекающей из условия оптимальности управления для задач $P_{\varepsilon}$.

Доказательство. Справедливость теоремы при $j=0$ доказана в [14]. Предположим, что теорема верна при $j=0, k-1,1 \leq k<n$.

Задача $\bar{P}_{k}$ однозначно разрешима и ее решение определяется равенствами (5), (10) при $j=\overline{0, k}$, и соотношениями, вытекающими из условия оптимальности управления, т. е.

$$
\frac{\partial \bar{H}_{k}}{\partial \bar{u}_{k}}=0, E_{1} \frac{d \bar{\psi}_{k}}{d t}=-\left(\frac{\partial \bar{H}_{k}}{\partial \bar{w}_{k}}\right),
$$

где

$$
\begin{gathered}
\bar{H}_{k}\left(\bar{v}_{k}, \bar{\psi}_{k}, t\right)= \\
=\left\langle\bar{\psi}_{k}, A_{0}(t) \bar{w}_{k}+B_{0}(t) \bar{u}_{k}+\right. \\
+\left[\widehat{\widehat{\Phi}}_{k-1}(t, \varepsilon)\right]_{k}-
\end{gathered}
$$

$$
\begin{gathered}
\left.-E_{2} d \bar{w}_{k-1} / d t-E_{3} d \bar{w}_{k-2} / d t\right\rangle- \\
-\left\langle\bar{w}_{k}, 1 / 2 W_{0}(t) \bar{w}_{k}+\left[\hat{\bar{\zeta}}_{k-1}(t, \varepsilon)\right]_{k}-\right. \\
\left.-E_{2} d \bar{\psi}_{k-1} / d t-E_{3} d \bar{\psi}_{k-2} / d t\right\rangle- \\
-\left\langle\bar{u}_{k}, 1 / 2 R_{0}(t) \bar{u}_{k}+\left[\hat{\bar{\varrho}}_{k-1}(t, \varepsilon)\right]_{k}\right\rangle .
\end{gathered}
$$

Соотношения (25) совпадают с (18), (19) при $j=k$ с учетом $\bar{\xi}_{j}(t)=\bar{\psi}_{j}(t), j=\overline{0, k}$. Принимая во внимание равенства $E_{1} Q_{0 k} \xi(0)=E_{1} Q_{0(k-1)} \psi(0)$,

$E_{1} Q_{1 k} \xi(0)=E_{1} Q_{1(k-2)} \psi(0), \quad \bar{\xi}_{k}(t)=\bar{\psi}_{k}(t)$, получаем совпадение условия $E_{1} \bar{\psi}_{k}(T)=-E_{1}\left(Q_{0(k-1)} \psi(0)+Q_{1(k-2)} \psi(0)\right) \quad$ для сопряженной переменной с равенством из (24) при $j=k$ после применения к нему $E_{1}$.

Предположим, что при $j<k$ справедливы соотношения

$$
\begin{gathered}
\bar{\xi}_{\mathrm{j}}(t)=\bar{\psi}_{j}(t), E_{1} \Pi_{0(j+1)} \xi\left(\tau_{0}\right)+ \\
+\left(E_{2}+E_{3}\right) \Pi_{0 j} \xi\left(\tau_{0}\right)=\Pi_{0 j} \psi\left(\tau_{0}\right), \\
E_{1} \Pi_{1(j+2)} \xi\left(\tau_{1}\right)+E_{2} \Pi_{1(j+1)} \xi\left(\tau_{1}\right)+ \\
E_{3} \Pi_{1 j} \xi\left(\tau_{1}\right)=\Pi_{1 j} \psi\left(\tau_{1}\right), \\
E_{1} Q_{0(j+1)} \xi\left(\sigma_{0}\right)+\left(E_{2}+\right. \\
\left.+E_{3}\right) Q_{0 j} \xi\left(\sigma_{0}\right)=Q_{0 j} \psi\left(\sigma_{0}\right), \\
E_{1} Q_{1(j+2)} \xi\left(\sigma_{1}\right)+E_{2} Q_{1(j+1)} \xi\left(\sigma_{1}\right)+ \\
+E_{3} Q_{1 j} \xi\left(\sigma_{1}\right)=Q_{1 j} \psi\left(\sigma_{1}\right) .
\end{gathered}
$$

Принимая во внимание равенства $E_{1} \Pi_{i 0} \xi\left(\tau_{i}\right)=E_{1} \Pi_{11} \xi\left(\tau_{1}\right)=0$,

$E_{1} Q_{i 0} \xi\left(\sigma_{i}\right)=E_{1} Q_{11} \xi\left(\sigma_{1}\right)=0, \quad E_{2} \Pi_{10} \xi\left(\tau_{1}\right)=0$, $E_{2} Q_{10} \xi\left(\sigma_{1}\right)=0$, которые следуют из (21), (23) при $i=0,1, j=0$ и $i=1, j=1$ в силу свойства пограничных функций на бесконечности, учитывая однозначную разрешимость задач $\prod_{i k} P, Q_{i k} P, i=0,1$, а также (26), получаем совпадение условий оптимальности управления задач $\prod_{i k} P, Q_{i k} P, i=0,1$, с соотношениями (20)-(23) аналогично задаче $\bar{P}_{k}$. Дополнительные пояснения требуются только для краевых условий задач $Q_{i k} P, i=0,1$, которые имеют вид

$$
\begin{aligned}
\left(E_{1}+E_{2}\right) & Q_{0 k} \psi(0)=-E_{2}\left(\bar{\psi}_{k}(t)+\right. \\
& \left.+Q_{1(k-1)} \psi(0)\right), \\
Q_{1 k} \psi(0)= & -E_{3}\left(\bar{\psi}_{k}(t)+Q_{0 k} \psi(0)\right) .
\end{aligned}
$$


Приближения любого порядка асимптотического решения...

Легко заметить, что при выполнении условий (26), равенстве $\bar{\xi}_{k}(T)=\bar{\psi}_{k}(T)$, краевое условие (27) совпадает с (24) при $j=k$ после применения к нему $E_{2}$, а (28) совпадает с (24) с $j=k$ после применения к нему $E_{3}$.

Теорема доказана.

Теорема 2. Сумма критериев качества $\bar{J}_{j}+\Pi_{1(j-1)} J+Q_{1(j-1)} J$ в задачах $\bar{P}_{j}, \Pi_{1(j-1)} P$, $Q_{1(j-1)} P$ получается в результате преобразования коэффициента $J_{2 j}$ из разложения (13), а сумма критериев качества $\Pi_{0 j} J+Q_{0 j} J$ в задачах $\Pi_{0 j} P, Q_{0 j} P$ получается в результате преобразования коэфбициента $J_{2 j+1}$ из разложения (13).

Доказательство. Преобразование коэффициентов $J_{j}, j=\overline{0,2}$, приведено в [14]. Предположим, что найдено решение задач $\bar{P}_{j}, \Pi_{0 j} P, Q_{0 j} P, \Pi_{1(j-1)} P, Q_{1(j-1)} P, j=\overline{0, n-1}$.

Докажем теорему для коэффициента $J_{2 n}$. Представим его в виде суммы пяти слагаемых и преобразуем каждое из них при помощи условий оптимальности управления уже построенных задач оптимального управления и формулы интегрирования по частям.

$$
\begin{gathered}
\int_{0}^{T} \bar{F}_{2 n}(t) d t+\int_{0}^{+\infty} \Pi_{0(2 n-1)} F\left(\tau_{0}\right) d \tau_{0}+ \\
+\int_{-\infty}^{0} Q_{0(2 n-1)} F\left(\sigma_{0}\right) d \sigma_{0}+\int_{0}^{+\infty} \Pi_{1(2 n-2)} F\left(\tau_{1}\right) d \tau_{1}+ \\
+\int_{-\infty}^{0} Q_{1(2 n-2)} F\left(\sigma_{1}\right) d \sigma_{1} .
\end{gathered}
$$

Введем обозначение

$$
\begin{gathered}
\Delta_{j} \omega(t, \varepsilon), \omega(t, \varepsilon)=\left(v(t, \varepsilon)^{\prime}, \xi(t, \varepsilon)^{\prime}\right)^{\prime}: \\
\Delta_{j} \omega(t, \varepsilon)=\omega(t, \varepsilon)-\widetilde{\bar{\omega}}_{j-1}(t, \varepsilon)- \\
-\sum_{i=0}^{1}\left(\tilde{\Pi}_{i(j-1)} \omega\left(\tau_{i}, \varepsilon\right)+\tilde{Q}_{i(j-1)} \omega\left(\sigma_{i}, \varepsilon\right)\right)= \\
=\Delta_{j} \bar{\omega}(t, \varepsilon)+ \\
+\sum_{i=0}^{1}\left(\Delta_{j} \Pi_{i} \omega\left(\tau_{i}, \varepsilon\right)+\Delta_{j} Q_{i} \omega\left(\sigma_{i}, \varepsilon\right)\right),
\end{gathered}
$$

где

$$
\begin{gathered}
\Delta_{j} \bar{\omega}(t, \varepsilon)=\bar{\omega}(t, \varepsilon)- \\
-\widetilde{\bar{\omega}}_{j-1}(t, \varepsilon)=\varepsilon^{j} \bar{\omega}_{j}(t)+\alpha\left(\varepsilon^{j+1}\right), \\
\Delta_{j} \Pi_{i} \omega\left(\tau_{i}, \varepsilon\right)=\Pi_{i} \omega\left(\tau_{i}, \varepsilon\right)- \\
-\tilde{\Pi}_{i(j-1)} \omega\left(\tau_{i}, \varepsilon\right)=\varepsilon^{j} \Pi_{i j} \omega\left(\tau_{i}\right)+\alpha\left(\varepsilon^{j+1}\right),
\end{gathered}
$$

$$
\begin{gathered}
\Delta_{j} Q_{i} \omega\left(\sigma_{i}, \varepsilon\right)=Q_{i} \omega\left(\sigma_{i}, \varepsilon\right)-\tilde{Q}_{i(j-1)} \omega\left(\sigma_{i}, \varepsilon\right)= \\
=\varepsilon^{j} Q_{i j} \omega\left(\sigma_{i}\right)+\alpha\left(\varepsilon^{j+1}\right), i=0,1 .
\end{gathered}
$$

После решения задач $\bar{P}_{j}, j=\overline{0, n-1}$, неизвестным в $\bar{F}_{2 n}(t)$ является выражение

$$
\begin{aligned}
& \left\langle\bar{w}_{n}, 1 / 2 W_{0}(t) \bar{w}_{n}+\left[W(t, \varepsilon) \widetilde{\bar{w}}_{n-1}(t, \varepsilon)\right]_{n}\right\rangle+ \\
& +\left\langle\bar{u}_{n}, 1 / 2 R_{0}(t) \bar{u}_{n}+\left[R(t, \varepsilon) \widetilde{\bar{u}}_{n-1}(t, \varepsilon)\right]_{n}\right\rangle+ \\
& +\left[\left\langle\Delta_{n+1} \bar{w}(t, \varepsilon),\left\{W(t, \varepsilon) \widetilde{\bar{w}}_{n-1}(t, \varepsilon)\right\}_{n-1}\right\rangle\right]_{2 n}+ \\
& +\left[\left\langle\Delta_{n+1} \bar{u}(t, \varepsilon),\left\{R(t, \varepsilon) \widetilde{\bar{u}}_{n-1}(t, \varepsilon)\right\}_{n-1}\right\rangle\right]_{2 n} .
\end{aligned}
$$

Подставляя $\Delta_{j} \bar{\omega}(t, \varepsilon)$ в (2), приравнивая коэффициенты при одинаковых степенях $\varepsilon$, зависящие от $t$, получаем уравнение

$$
\begin{gathered}
\mathcal{E}(\varepsilon)\left(\frac{d \Delta_{j} \bar{w}(t, \varepsilon)}{d t}+\frac{d \widetilde{\bar{w}}_{j-1}(t, \varepsilon)}{d t}\right)= \\
=A(t, \varepsilon) \Delta_{j} \bar{w}(t, \varepsilon)+ \\
+B(t, \varepsilon) \Delta_{j} \bar{u}(t, \varepsilon)+\left[\widehat{\bar{\Phi}}_{j-1}(t, \varepsilon)\right]_{j} .
\end{gathered}
$$

В силу теоремы 1 из (18), (19), следуют равенства

$$
\begin{gathered}
\left\{R(t, \varepsilon) \widetilde{\bar{u}}_{j}(t, \varepsilon)\right\}_{j}=\left\{B(t, \varepsilon)^{\prime} \widetilde{\bar{\psi}}_{j}(t, \varepsilon)\right\}_{j}, \\
\left\{W(t, \varepsilon) \widetilde{\bar{w}}_{j}(t, \varepsilon)\right\}_{j}= \\
=\left\{\mathcal{E}(\varepsilon) \frac{d \widetilde{\bar{\psi}}_{j}(t, \varepsilon)}{d t}\right\}_{j}+\left\{A(t, \varepsilon)^{\prime} \widetilde{\bar{\psi}}_{j}(t, \varepsilon)\right\}_{j} .
\end{gathered}
$$

Преобразуем интеграл

$$
\begin{aligned}
& \int_{0}^{T}\left(\left[\left\langle\Delta_{n+1} \bar{w},\left\{W(t, \varepsilon) \widetilde{\bar{w}}_{n-1}(t, \varepsilon)\right\}_{n-1}\right\rangle\right]_{2 n}+\right. \\
& \left.+\left[\left\langle\Delta_{n+1} \bar{u},\left\{R(t, \varepsilon) \widetilde{\bar{u}}_{n-1}(t, \varepsilon)\right\}_{n-1}\right\rangle\right]_{2 n}\right) d t
\end{aligned}
$$

из $\int_{0}^{T} \bar{F}_{2 n}(t) d t$, используя (30), (31) при $j=n-1$.

$$
\begin{gathered}
\int_{0}^{T}\left(\left\langle\left\langle\Delta_{n+1} \bar{w},\left\{\mathcal{E}(\varepsilon) \frac{d \widetilde{\bar{\psi}}_{n-1}}{d t}\right\}_{n-1}\right\rangle\right]_{2 n}+\right. \\
+\left[\left\langle\widetilde{\bar{\psi}}_{n-1}, A(t, \varepsilon) \Delta_{n+1} \bar{w}+\right.\right. \\
\left.\left.\left.+B(t, \varepsilon) \Delta_{n+1} \bar{u}\right\rangle\right]_{2 n}\right) d t= \\
=\int_{0}^{T}\left(\left\langle\Delta_{n+1} \bar{w},\left\{\mathcal{E}(\varepsilon) \frac{d \widetilde{\bar{\psi}}_{n-1}}{d t}\right\}_{n-1}\right\rangle\right]_{2 n}+
\end{gathered}
$$


М. А. Калачникова, Г. А. Курина

$$
\begin{gathered}
+\left[\left\langle\widetilde{\bar{\psi}}_{n-1}, \mathcal{E}(\varepsilon) \frac{d \Delta_{n+1} \bar{w}}{d t}-\right.\right. \\
-A(t, \varepsilon) \widetilde{\bar{w}}_{n}-B(t, \varepsilon) \widetilde{\bar{u}}_{n}-\widehat{\bar{f}}(t, \varepsilon)+ \\
\left.\left.\left.+\mathcal{E}(\varepsilon) \frac{d \widetilde{\bar{w}}_{j-1}}{d t}\right\rangle\right]_{2 n}\right) d t
\end{gathered}
$$

Интегрируя по частям последнее выражение с учетом равенства $\Delta_{n+1} \bar{w}=\Delta_{n} \bar{w}-\varepsilon^{n} \bar{w}_{n}$, отбрасывая известные слагаемые, в результате имеем

$$
\begin{gathered}
{\left.\left[\left\langle\left(1 / \varepsilon E_{1}+E_{2}+\varepsilon E_{3}\right) \Delta_{n} \bar{w}, \widetilde{\bar{\psi}}_{n-1}\right\rangle\right]_{2 n-1}\right|_{0} ^{T}-} \\
\quad-\int_{0}^{T}\left(\left\langle\bar{w}_{n},\left[A(t, \varepsilon)^{\prime} \widetilde{\bar{\psi}}_{n-1}\right]_{n}+\right.\right. \\
\left.+E_{2} d \bar{\psi}_{n-1} / d t+E_{3} d \bar{\psi}_{n-2} / d t\right\rangle+ \\
\left.+\left\langle\bar{u}_{n},\left[B(t, \varepsilon)^{\prime} \widetilde{\bar{\psi}}_{n-1}\right]_{n}\right\rangle\right) d t
\end{gathered}
$$

$T$ Подставляя последнее выражение в $\int_{0}^{T} \bar{F}_{2 n}(t) d t$, получаем

$$
\begin{gathered}
{\left.\left[\left\langle\Delta_{n} \bar{w},\left(1 / \varepsilon E_{1}+E_{2}+\varepsilon E_{3}\right) \widetilde{\widetilde{\psi}}_{n-1}\right\rangle\right]_{2 n-1}\right|_{0} ^{T}+} \\
+\bar{J}_{n}-\left\langle\bar{w}_{n}(T), E_{1}\left(Q_{0(n-1)} \psi(0)+\right.\right. \\
\left.\left.+Q_{1(n-2)} \psi(0)\right)\right\rangle .
\end{gathered}
$$

После решения задач $\bar{P}_{j}, \Pi_{0 j} P, j=\overline{0, n-1}$, неизвестным в $\Pi_{0(2 n-1)} F\left(\tau_{0}\right)$ является

$$
\begin{aligned}
& {\left[\left\langle\Delta_{n} \Pi_{0} w,\left\{W\left(\varepsilon \tau_{0}, \varepsilon\right) \widetilde{\bar{w}}_{n-1}\left(\varepsilon \tau_{0}, \varepsilon\right)\right\}_{n-1}\right\rangle\right]_{2 n-1}+} \\
+ & {\left[\left\langle\Delta_{n} \Pi_{0} u,\left\{R\left(\varepsilon \tau_{0}, \varepsilon\right) \widetilde{\bar{u}}_{n-1}\left(\varepsilon \tau_{0}, \varepsilon\right)\right\}_{n-1}\right\rangle\right]_{2 n-1}+} \\
+ & {\left[\left\langle\Delta_{n} \bar{w}\left(\varepsilon \tau_{0}, \varepsilon\right),\left\{W\left(\varepsilon \tau_{0}, \varepsilon\right) \tilde{\Pi}_{0(n-1)} w\right\}_{n-1}\right\rangle\right]_{2 n-1}+} \\
+ & {\left[\left\langle\Delta_{n} \Pi_{0} w,\left\{W\left(\varepsilon \tau_{0}, \varepsilon\right) \tilde{\Pi}_{0(n-1)} w\right\}_{n-1}\right\rangle\right]_{2 n-1}+} \\
+ & {\left[\left\langle\Delta_{n} \bar{u}\left(\varepsilon \tau_{0}, \varepsilon\right),\left\{R\left(\varepsilon \tau_{0}, \varepsilon\right) \tilde{\Pi}_{0(n-1)} u\right\}_{n-1}\right\rangle\right]_{2 n-1}+} \\
& +\left[\left\langle\Delta_{n} \Pi_{0} u,\left\{R\left(\varepsilon \tau_{0}, \varepsilon\right) \tilde{\Pi}_{0(n-1)} u\right\}_{n-1}\right\rangle\right]_{2 n-1} .
\end{aligned}
$$

Преобразуем интеграл по промежутку $[0,+\infty)$ от последнего выражения. Покажем, что он имеет вид

$$
\begin{gathered}
-\left[\left\langle\Delta_{n} \Pi_{0} w(0, \varepsilon),\left(1 / \varepsilon E_{1}+\right.\right.\right. \\
\left.\left.\left.\left.+E_{2}+\varepsilon E_{3}\right) \widetilde{\bar{\psi}}_{n-1}(0)\right)\right\rangle\right]_{2 n-1}- \\
-\left[\left\langle\Delta_{n} \bar{w}(0, \varepsilon),\left(E_{1}+\right.\right.\right.
\end{gathered}
$$

$$
\begin{gathered}
\left.\left.\left.+E_{2}+\varepsilon E_{3}\right) \tilde{\Pi}_{0(n-1)} \psi(0, \varepsilon)\right\rangle\right]_{2 n-1}- \\
-\left[\left\langle\Delta_{n} \Pi_{0} w(0, \varepsilon),\left(E_{1}+E_{2}+\right.\right.\right. \\
\left.\left.\left.+\varepsilon E_{3}\right) \tilde{\Pi}_{0(n-1)} \psi(0, \varepsilon)\right\rangle\right]_{2 n-1} .
\end{gathered}
$$

Подставляя $\Delta_{j} \Pi_{0} \omega\left(\tau_{0}, \varepsilon\right)$ в $(2)$, приравнивая коэффициенты при одинаковых степенях $\varepsilon$, зависящие от $\tau_{0}$, получаем уравнение

$$
\begin{gathered}
\left(\frac{1}{\varepsilon} E_{1}+E_{2}+\varepsilon E_{3}\right)\left(\frac{d \Delta_{j} \Pi_{0} w}{d \tau_{0}}+\right. \\
\left.+\frac{d \tilde{\Pi}_{0(j-1)} w}{d \tau_{0}}\right)=A\left(\varepsilon \tau_{0}, \varepsilon\right) \Delta_{j} \Pi_{0} w+ \\
+B\left(\varepsilon \tau_{0}, \varepsilon\right) \Delta_{j} \Pi_{0} u+\left[\hat{\Pi}_{0(j-1)} \Phi\left(\tau_{0}, \varepsilon\right)\right]_{j} .
\end{gathered}
$$

В силу теоремы 1 из (20), (21) при $i=0$ получаем соотношения

$$
\begin{gathered}
\left\{R\left(\varepsilon \tau_{0}, \varepsilon\right) \tilde{\Pi}_{0 j} u\left(\tau_{0}, \varepsilon\right)\right\}_{j}= \\
=\left\{B\left(\varepsilon \tau_{0}, \varepsilon\right)^{\prime}\left(\varepsilon E_{1}+E_{2}+E_{3}\right) \tilde{\Pi}_{0 j} \psi\left(\tau_{0}, \varepsilon\right)\right\}_{j}, \\
\left\{W\left(\varepsilon \tau_{0}, \varepsilon\right) \tilde{\Pi}_{0 j} w\left(\tau_{0}, \varepsilon\right)\right\}_{j}= \\
=\left\{\left(E_{1}+E_{2}+\varepsilon E_{3}\right) \frac{d \tilde{\Pi}_{0 j} \psi\left(\tau_{0}, \varepsilon\right)}{d \tau_{0}}\right\}_{j}+ \\
+\left\{A\left(\varepsilon \tau_{0}, \varepsilon\right)^{\prime}\left(\varepsilon E_{1}+E_{2}+E_{3}\right) \tilde{\Pi}_{0 j} \psi\left(\tau_{0}, \varepsilon\right)\right\}_{j} .
\end{gathered}
$$

Сначала преобразуем интеграл по промежутку $[0,+\infty)$ от первых двух слагаемых в $\Pi_{0(2 n-1)} F\left(\tau_{0}\right)$ с учетом условий оптимальности управления (30), (31) при $j=n-1$, уравнения (32) при $j=n$, формулы интегрирования по частям. Интеграл по промежутку $[0,+\infty)$ от остальных слагаемых в $\prod_{0(2 n-1)} \mathrm{F}\left(\tau_{0}\right)$ будем преобразовывать, используя (33), (34) при $j=n-1$, уравнения (29) и (31) при $j=n$ и формулу интегрирования по частям. Отбросим известные слагаемые с учетом замечания. В итоге получаем вышеуказанное представление для $\int_{0}^{+\infty} \Pi_{0(2 n-1)} F\left(\tau_{0}\right) d \tau_{0}$.

$$
\begin{gathered}
\int_{-\infty}^{0} Q_{0(2 n-1)}^{\text {Преобразуя }} F\left(\sigma_{0}\right) d \sigma_{0}, \text { получогичным образом } \\
{\left[\left\langle\widetilde{\bar{\psi}}_{n-1}(T, \varepsilon),\left(1 / \varepsilon E_{1}+E_{2}+\right.\right.\right.} \\
\left.\left.\left.+\varepsilon E_{3}\right) \Delta_{n} Q_{0} w(0, \varepsilon)\right\rangle\right]_{2 n-1}+\left[\left\langle\Delta_{n} \bar{w}(T, \varepsilon)+\right.\right. \\
+\Delta_{n} Q_{0} w(0, \varepsilon),\left(E_{1}+\right.
\end{gathered}
$$


Приближения любого порядка асимптотического решения...

$\left.\left.\left.+E_{2}+\varepsilon E_{3}\right) \tilde{Q}_{0(n-1)} \psi(0, \varepsilon)\right\rangle\right]_{2 n-1}$.

После решения задач $\bar{P}_{j}, \Pi_{0 j} P, Q_{0 j} P$, $\Pi_{1(j-1)} P, Q_{1(j-1)} P, j=\overline{0, n-1}$, неизвестным в $\Pi_{1(2 n-2)} F\left(\tau_{1}\right)$ является

$$
\begin{aligned}
& {\left[\left\langle\Delta_{n-1} \Pi_{1} w,\left\{W ( \varepsilon ^ { 2 } \tau _ { 1 } , \varepsilon ) \left(\tilde{\bar{w}}_{n-1}\left(\varepsilon^{2} \tau_{1}, \varepsilon\right)+\right.\right.\right.\right.}\left.\left.\left.\left.+\tilde{\Pi}_{0(n-1)} w\left(\varepsilon \tau_{1}, \varepsilon\right)\right)\right\}_{n-1}\right\rangle\right]_{2 n-2}+ \\
&+\left[\left\langle\Delta_{n-1} \Pi_{1} u,\left\{R ( \varepsilon ^ { 2 } \tau _ { 1 } , \varepsilon ) \left(\tilde{\bar{u}}_{n-1}\left(\varepsilon^{2} \tau_{1}, \varepsilon\right)+\right.\right.\right.\right. \\
&\left.\left.\left.\left.+\tilde{\Pi}_{0(n-1)} u\left(\varepsilon \tau_{1}, \varepsilon\right)\right)\right\}_{n-1}\right\rangle\right]_{2 n-2}+ \\
&+\left[\left\langle\Delta_{n} \bar{w}\left(\varepsilon^{2} \tau_{1}, \varepsilon\right)+\Delta_{n} \Pi_{0} w\left(\varepsilon \tau_{1}, \varepsilon\right)+\right.\right. \\
&\left.\left.+\Delta_{n} \Pi_{1} w,\left\{W\left(\varepsilon^{2} \tau_{1}, \varepsilon\right) \tilde{\Pi}_{1(n-2)} w\left(\tau_{1}, \varepsilon\right)\right\}_{n-2}\right\rangle\right]_{2 n-2}+ \\
&+\left[\left\langle\left\langle\Delta_{n} \bar{u}\left(\varepsilon^{2} \tau_{1}, \varepsilon\right)+\Delta_{n} \Pi_{0} u\left(\varepsilon \tau_{1}, \varepsilon\right)+\right.\right.\right. \\
&\left.\left.+\Delta_{n} \Pi_{1} u,\left\{R\left(\varepsilon^{2} \tau_{1}, \varepsilon\right) \tilde{\Pi}_{1(n-2)} u\left(\tau_{1}, \varepsilon\right)\right\}_{n-2}\right\rangle\right]_{2 n-2}+ \\
&+\left\langle\Pi_{1(n-1)} w, 1 / 2 W_{0}(0) \Pi_{1(n-1)} w+\right. \\
&\left.+\left[W\left(\varepsilon^{2} \tau_{1}, \varepsilon\right) \tilde{\Pi}_{1(n-2)} w\right]_{n-1}\right\rangle+ \\
&+\left\langle\Pi_{1(n-1)} u, 1 / 2 R_{0}(0) \Pi_{1(n-1)} u+\right. \\
&\left.+\left[R\left(\varepsilon^{2} \tau_{1}, \varepsilon\right) \tilde{\Pi}_{1(n-2)} u\right]_{n-1}\right\rangle .
\end{aligned}
$$

Подставляя $\Delta_{n} \Pi_{1} \omega\left(\tau_{1}, \varepsilon\right)$ в (2), приравнивая коэффициенты, зависящие от $\tau_{1}$, имеем

$$
\begin{gathered}
\left(\frac{1}{\varepsilon^{2}} E_{1}+\frac{1}{\varepsilon} E_{2}+E_{3}\right)\left(\frac{d \Delta_{j} \Pi_{1} w\left(\tau_{1}, \varepsilon\right)}{d \tau_{1}}+\right. \\
\left.+\frac{d \tilde{\Pi}_{1(j-1)} w\left(\tau_{1}, \varepsilon\right)}{d \tau_{1}}\right)= \\
=A\left(\varepsilon^{2} \tau_{1}, \varepsilon\right) \Delta_{j} \Pi_{1} w\left(\tau_{1}, \varepsilon\right)+ \\
+B\left(\varepsilon^{2} \tau_{1}, \varepsilon\right) \Delta_{j} \Pi_{1} u\left(\tau_{1}, \varepsilon\right)+\left[\hat{\Pi}_{1(j-1)} \Phi\left(\tau_{1}, \varepsilon\right)\right]_{j} .
\end{gathered}
$$

В силу теоремы 1 из (20), (21) при $i=1$ следуют соотношения

$$
\begin{gathered}
\left\{R\left(\varepsilon^{2} \tau_{1}, \varepsilon\right) \tilde{\Pi}_{1 j} u\left(\tau_{1}, \varepsilon\right)\right\}_{j}= \\
=\left\{B\left(\varepsilon^{2} \tau_{1}, \varepsilon\right)^{\prime}\left(\varepsilon^{2} E_{1}+\varepsilon E_{2}+E_{3}\right) \tilde{\Pi}_{1 j} \psi\left(\tau_{1}, \varepsilon\right)\right\}_{j}, \\
\left\{W\left(\varepsilon^{2} \tau_{1}, \varepsilon\right) \tilde{\Pi}_{1 j} w\left(\tau_{1}, \varepsilon\right)\right\}_{j}= \\
=\left\{\frac{d \tilde{\Pi}_{1 j} \psi\left(\tau_{1}, \varepsilon\right)}{d \tau_{1}}\right\}_{j}+\left\{A ( \varepsilon ^ { 2 } \tau _ { 1 } , \varepsilon ) ^ { \prime } \left(\varepsilon^{2} E_{1}+\right.\right. \\
\left.\left.+\varepsilon E_{2}+E_{3}\right) \tilde{\Pi}_{1 j} \psi\left(\tau_{1}, \varepsilon\right)\right\}_{j} .
\end{gathered}
$$

Преобразуем интеграл по промежутку $[0,+\infty)$ от первых четырех слагаемых в $\int_{1(2 n-2)}^{+\infty} F\left(\tau_{1}\right) d \tau_{1}$, используя сначала (30), (31), (35) при $j=n-1$, далее (33), (34), (35) при $j=n-1$, а затем (36), (37) при $j=n-2$ и (29), (32), (35) при $j=n$, а также формулу интегрирования по частям. Учитывая замечание, отбросим известные слагаемые и подставим полученное выражение в $\int_{0}^{+\infty} \Pi_{1(2 n-2)} F\left(\tau_{1}\right) d \tau_{1}$. В результате для $\int_{0}^{+\infty} \Pi_{1(2 n-2)} F\left(\tau_{1}\right) d \tau_{1}$ имеем выражение

$$
\begin{gathered}
-\left[\left\langle\Delta_{n-1} \Pi_{1} w(0, \varepsilon),\left(1 / \varepsilon^{2} E_{1}+1 / \varepsilon E_{2}+\right.\right.\right. \\
\left.\left.\left.+E_{3}\right) \widetilde{\bar{\psi}}_{n-1}(0, \varepsilon)\right\rangle\right]_{2 n-2}- \\
-\left[\left\langle\Delta_{n-1} \Pi_{1} w(0, \varepsilon),\left(1 / \varepsilon^{2} E_{1}+1 / \varepsilon E_{2}+\right.\right.\right. \\
\left.\left.\left.+E_{3}\right) \tilde{\Pi}_{0(n-1)} \psi(0, \varepsilon)\right\rangle\right]_{2 n-2}- \\
-\left[\left\langle\Delta_{n} \bar{w}(0, \varepsilon)+\Delta_{n} \Pi_{0} w(0, \varepsilon)+\right.\right. \\
\left.\left.+\Delta_{n} \Pi_{1} w(0, \varepsilon), \tilde{\Pi}_{1(n-2)} \psi(0, \varepsilon)\right\rangle\right]_{2 n-2}+\Pi_{1(n-1)} J . \\
\text { Преобразуя } \int_{-\infty}^{0} Q_{1(2 n-1)} F\left(\sigma_{1}\right) d \sigma_{1} \text { аналогич- }
\end{gathered}
$$

ным образом, получаем

$$
\left[\left\langle\left(1 / \varepsilon^{2} E_{1}+\right.\right.\right.
$$

$$
\begin{gathered}
\left.\left.\left.+1 / \varepsilon E_{2}\right) \Delta_{n-1} Q_{1} w(0, \varepsilon), \widetilde{\bar{\psi}}_{n-1}(T, \varepsilon)\right\rangle\right]_{2 n-2}+ \\
+\left[\left\langle E_{3} \Delta_{n-1} Q_{1} w(0, \varepsilon), \widetilde{\bar{\psi}}_{n-2}(T, \varepsilon)\right\rangle\right]_{2 n-2}+ \\
+\left[\left\langle\left(1 / \varepsilon^{2} E_{1}+\right.\right.\right. \\
\left.\left.\left.+1 / \varepsilon E_{2}\right) \Delta_{n-1} Q_{1} w(0, \varepsilon), \tilde{Q}_{0(n-1)} \psi(0, \varepsilon)\right\rangle\right]_{2 n-2}+ \\
+\left[\left\langle E_{3} \Delta_{n-1} Q_{1} w(0, \varepsilon), \tilde{Q}_{0(n-2)} \psi(0, \varepsilon)\right\rangle\right]_{2 n-2}+ \\
+\left[\left\langle\Delta_{n} \bar{w}(T, \varepsilon)+\Delta_{n} Q_{0} w(0, \varepsilon)+\right.\right. \\
\left.\left.+\Delta_{n} Q_{1} w(0, \varepsilon), \tilde{Q}_{1(n-2)} \psi(0, \varepsilon)\right\rangle\right]_{2 n-2}+Q_{1(n-1)} J .
\end{gathered}
$$

Далее преобразуем внеинтегральные члены. Соотношение для начальных условий $w(t, \varepsilon)$, вытекающее из (3), имеет вид

$$
\begin{gathered}
\Delta_{j} \bar{w}(0, \varepsilon)+\widetilde{\bar{w}}_{j-1}(0, \varepsilon)+ \\
+\sum_{i=0}^{1}\left(\Delta_{n} \Pi_{i} w(0, \varepsilon)+\right. \\
+\tilde{\Pi}_{i(n-1)} \psi(0, \varepsilon)+\Delta_{n} Q_{i} w\left(-T / \varepsilon^{i+1}, \varepsilon\right)+ \\
\left.+\tilde{Q}_{i(n-1)} \psi\left(-T / \varepsilon^{i+1}, \varepsilon\right)\right)=w^{0} .
\end{gathered}
$$


Учитывая теорему 1, из (24) получаем краевые условия для сопряженных переменных

$$
\begin{gathered}
\bar{\psi}_{j}(T)+E_{1}\left(Q_{0(j-1)} \psi(0)+Q_{1(j-2)} \psi(0)\right)+ \\
+E_{2}\left(Q_{0 j} \psi(0)+Q_{1(j-1)} \psi(0)\right)+ \\
+E_{3}\left(Q_{0 j} \psi(0)+Q_{1 j} \psi(0)\right)=0 .
\end{gathered}
$$

Суммируя выражения, полученные в результате преобразования пяти слагаемых в $J_{2 n}$, используя (38), (39), а также равенства $\Delta_{n} \Pi_{1} w\left(\tau_{1}, \varepsilon\right)=\Delta_{n-1} \Pi_{1} w\left(\tau_{1}, \varepsilon\right)-\varepsilon^{n+1} \Pi_{1(n-1)} w\left(\tau_{1}\right)$, $\Delta_{n} Q_{1} w\left(\sigma_{1}, \varepsilon\right)=\Delta_{n-1} Q_{1} w\left(\sigma_{1}, \varepsilon\right)-\varepsilon^{n+1} Q_{1(n-1)} w\left(\sigma_{1}\right)$, после отбрасывания известных слагаемых с учетом замечания, получаем сумму критериев качества $\bar{J}_{n}+\Pi_{1(n-1)} J+Q_{1(n-1)} J$, откуда следует утверждение теоремы для $J_{2 n}$.

Доказательство для $J_{2 n+1}$ проводится аналогичным образом.

Теорема доказана.

\section{4. ПРИМЕР}

Для иллюстрации эффективности метода прямой схемы приведем таблицу значений функционала при $\varepsilon=0.25$ для следующей линейно-квадратичной задачи:

$$
\begin{gathered}
J(u)=\int_{0}^{1}\left(u^{2}+t u+y+z\right) d t \rightarrow \min , \\
d x / d t=x+u, x(0)=10, \\
\varepsilon d y / d t=-y+u, y(0)=10, \\
\varepsilon^{2} d z / d t=-y-u, z(0)=10 .
\end{gathered}
$$

В таблице ниже даны значения функционала, вычисленные при оптимальном управлении $u_{*}(t, \varepsilon)$ и его приближениях, а именно, $\bar{u}_{0}(t)$, которое является решением вырожденной задачи, получающейся из исходной при нулевом значении малого параметра, а также при приближении нулевого порядка $\tilde{u}_{0}(t, \varepsilon)$, включающем пограничные функции.

\begin{tabular}{|c|c|c|c|}
\hline$\varepsilon$ & $J\left(\bar{u}_{0}\right)$ & $J\left(\tilde{u}_{0}\right)$ & $J\left(u_{*}\right)$ \\
\hline 0.25 & 3.061 & 3.047 & 3.047 \\
\hline
\end{tabular}

Из таблицы видно, что при переходе от управления вырожденной задачи к приближению оптимального управления нулевого порядка получается меньшее значение функционала.

\section{ЗАКЛЮЧЕНИЕ}

Метод прямой схемы является эффективным способом решения сингулярно возмущенных задач оптимального управления и может быть использован как в теории, так и при решении конкретных прикладных задач. Для построенного асимптотического приближения решения могут быть доказаны асимптотические оценки близости к точному решению и установлено невозрастание значений минимизируемого функционала при использовании следующего приближения оптимального управления.

Работа второго автора была поддержана Российским Научным Фондом (проект № 1711-01220).

\section{СПИСОК ЛИТЕРАТУРЫ}

1. Дмитриев, М. Г. Сингулярные возмущения в задачах управления / М. Г. Дмитриев, Г. А. Курина // Автоматика и телемеханика. 2006. - №1. - C. 3-51.

2. Zhang, Y. Singular perturbation and time scales in control theories and applications: an overview 2002-2012 / Y. Zhang, D. S. Naidu, C. Cai, Y. Zou // International Journal of Information and Systems Sciences. - 2014 - V. 9, No. 1. - P. 1-36.

3. Калашникова, М. А. Асимптотика решения трехтемповой задачи оптимального управления / М. А. Калашникова, Г. А. Курина // Труды XII всероссийского совещания по проблемам управления. ВСПУ. (Москва, 16-19 июня 2014 г.) - Москва, 2014. - С. 15601570.

4. Shishkin, G. Multiscale problems with various boundary layers for PDE'S in unbounded domains / G. Shishkin // 10th International Conference «Mathematical Modelling and Analysis» and 2nd International Conference "Computational Methods in Applied Mathematics». (Trakai, Lithuania, 1-5 June, 2005). - 2005. - P. 251-257.

5. Васильева, А. Б. Асимптотические разложения решений сингулярно возмущенных уравнений / А. Б. Васильева, В. Ф. Бутузов. Москва : Изд-во Наука, 1973. - 272 с. 
6. Воропаева, H. В. Геометрическая декомпозиция сингулярно возмущенных систем/ Н. В. Воропаева, Н. В. Соболев. - Москва : Изд-во Физматлит, 2009. - 356 с.

7. Kurina, G. A. Discrete singularly perturbed control problems (a survey). / G. A. Kurina, M. G. Dmitriev, D. S. Naidu // Dynamics of Continuous, Discrete and Impulsive Systems. Series B: Applications \& Algorithms. - 2017. - V. 24. P. 335-370.

8. Градштейн И. С. Дифференциальные уравнения, в которых множителем при производных входят различные степени малого параметра / И. С. Градштейн // Доклады АН CССР. - 1952. - Т. 82, № 1. - С. 5-8.

9. Тихонов А. Н. Системы дифференциальных уравнений, содержащие малые параметры при производных / А. Н. Тихонов // Математический сборник. - 1952. - Т. 31(73), № 3. - С. 575-586.

10. Васильева А. Б. Асимптотические формулы для решений систем обыкновенных дифференциальных уравнений, содержащих при производных параметры различных порядков малости / А. Б. Васильева // Доклады AH CCCP. - 1959. - Т. 128, № 6. - C. 1110-1113.

11. Khalil, H. K. Control of Linear Systems with Multiparameter Singular Perturbations /

Калашникова Маргарита Александровна ведущий консультант, Atos IT Solutions and Services, Воронеж.

Тел.: +7-904-211-5246

E-mail: margarita.kalashnikova@mail.ru

Курина Галина Алексеевна - д-р. физ.-мат. наук, профессор кафедры математического анализа Воронежского государственного университета, Воронежский экономико-правовой институт, Федеральный исследовательский центр «Информатика и управление» Российской академии наук.

Тел.: +7-4732-208690, +7-906-584-1966

E-mail: kurina@math.vsu.ru
H. K. Khalil, P. V. Kokotovic // Automatica. 1979. - V. 15, No. 2. - P. 197-207.

12. Mukaidani, H. Control of Deterministic and Stochastic Systems with Several Small Parameters - a Survey / H. Mukaidani, V. Dragan // Annals of the Academy of Romanian Scientists. Ser. Mathematics and its Applications. - 2009. -V. 1, No. 1. - P. 112-158.

13. Белокопьтов, С. В. Дмитриев М. Г. Решение классических задач оптимального управления с погранслоем / С. В. Белокопытов, М. Г. Дмитриев // Автоматика и телемеханика. - 1989. - № 7. - С. 71-82.

14. Калашникова, М. А. Асимптотика приближения нулевого порядка решения трехтемповой линейно-квадратичной задачи оптимального управления / М. А. Калашникова // Моделирование и анализ информационных систем. 2015. - Т. 22, № 1. - С. 85-104.

15. Kurina, G. A. High Order Asymptotic Solution of Linear-Quadratic Optimal Control Problems Under Cheap Control with Two Different Costs / G. A. Kurina, M. A. Kalashnikova // Proceeding of 21st International Conference on System Theory, Control and Computing. ICSTCC 2017. (Romania, Sinaia, 19-21 October 2017). - Romania, Sinaia, 2017. - P. 499-504.

Kalashnikova Margarita Aleksandrovna - Expert, Atos IT Solutions and Services, Voronezh. Tel.: +7-904-211-5246

E-mail: margarita.kalashnikova@mail.ru

Kurina Galina Alekseevna - Doctor of Sciences in Physics and Mathematics, professor of Mathematical Analysis Chair in Voronezh State University, Institute of Law and Economics, Federal Research Center "Computer Science and Control» of Russian Academy of Sciences.

Tel.: +7-4732-208690, +7-906-584-1966

E-mail: kurina@math.vsu.ru 